\title{
POLY-FREE CONSTRUCTIONS FOR RIGHT-ANGLED ARTIN GROUPS
}

\author{
SUSAN HERMILLER ${ }^{1}$ AND ZORAN ŠUNIḰ
}

\begin{abstract}
We show that every right-angled Artin group $A \Gamma$ defined by a graph $\Gamma$ of finite chromatic number is poly-free with poly-free length bounded between the clique number and the chromatic number of $\Gamma$. Further, a characterization of all right-angled Artin groups of poly-free length 2 is given, namely the group $A \Gamma$ has poly-free length 2 if and only if there exists an independent set of vertices $D$ in $\Gamma$ such that every cycle in $\Gamma$ meets $D$ at least twice. Finally, it is shown that $A \Gamma$ is a semidirect product of 2 free groups of finite rank if and only if $\Gamma$ is a finite tree or a finite complete bipartite graph. All of the proofs of the existence of poly-free structures are constructive.
\end{abstract}

\section{IntRoduction}

A group $G$ is poly-free if there exists a finite tower of subgroups

$$
1=G_{0} \unlhd G_{1} \unlhd \cdots \unlhd G_{N}=G
$$

for which each quotient $G_{i+1} / G_{i}$ is a free group. The least natural number $N$ for which such a tower exists is the poly-free length of $G$, denoted $\operatorname{pfl}(G)$. A group $G$ is poly-finitely-generated-free, or poly-fg-free, if there exists a tower of this form with the additional property that each of the quotients is a finitely generated free group. Since every map onto a free group splits, a poly-free group can be realized as an iterated semidirect product of free groups [15].

Examples of poly-fg-free groups include certain subgroups of Artin groups. Let $\Gamma$ be a finite simplicial graph; throughout the text we will assume that such graphs do not have loops or multiple edges. If the edges of $\Gamma$ are labeled by integers greater than one, the associated Artin group $A \Gamma$ has generators corresponding to the vertices, and relations

$$
\underbrace{a b a \cdots}_{n \text { letters }}=\underbrace{b a b \cdots}_{n \text { letters }}
$$

where $\{a, b\}$ is an edge of the graph labeled $n$. If, in addition, relations are added making each generator of order 2, the resulting quotient is a Coxeter group. Braid groups are the Artin groups whose Coxeter quotients are the symmetric groups. When the Coxeter quotient is finite, the Artin group is

Date: November 8, 2018.

Key words and phrases. Poly-free, right-angled Artin group, free group.

${ }^{1}$ Supported under NSF grant no. DMS-0071037 
said to be of finite type. Pure Artin groups are subgroups of Artin groups which are the kernel of the homomorphism onto the corresponding Coxeter group.

Pure braid groups are examples of poly-fg-free groups [1, as are pure finite type Artin groups whose Coxeter quotients are of type $B_{n}, D_{n}, I_{2}(p)$, and $F_{4}$ 4. If the graph associated to an Artin group is a tree, Hermiller and Meier [1] have shown that the Artin group is an extension of a finitely generated free group by the integers, and hence is poly-fg-free. Recently, Bestvina 2] has asked if all Artin groups of finite type, or indeed all Artin groups of any type, are virtually poly-free.

In this paper we investigate the poly-free properties of the class of rightangled Artin groups, which are the Artin groups for which the defining graph has every edge labeled 2. That is, for a graph $\Gamma$, the right-angled Artin group $A \Gamma$ is the group with generators in one-to-one correspondence with the set $V(\Gamma)$ of vertices of $\Gamma$, and relations $[v, w]=v w v^{-1} w^{-1}$, for each edge between vertices $v$ and $w$ of $\Gamma$. These groups are also known in the literature as graph groups, or free partially commutative groups. (See 7, [9], 10], 16] for information on normal forms for right-angled Artin groups and further references.)

Our main results are as follows.

Theorem A. Let $\Gamma$ be a finite graph or, more generally, a graph of finite chromatic number $\operatorname{chr}(\Gamma)$ and finite clique number clq $(\Gamma)$. The right-angled Artin group $A \Gamma$ is poly-free. Moreover,

$$
\operatorname{clq}(\Gamma) \leq \operatorname{pfl}(A \Gamma) \leq \operatorname{chr}(\Gamma),
$$

and there exists a poly-free tower for $A \Gamma$ of length $\operatorname{chr}(\Gamma)$.

During the preparation of the text, W. Dicks has pointed out to us that J. Howie 12 has established $|V(\Gamma)|$ as an upper bound for the poly-free length of a right-angled Artin group defined by a finite graph $\Gamma$. Thus the above result is an improvement in the case of finite graphs and a generalization to a class of infinite graphs.

A graph $\Gamma$ is said to have the doubly breakable cycle property if $\Gamma$ is not totally disconnected and there exists a vertex subset $D \subseteq V(\Gamma)$ such that the full subgraph of $\Gamma$ induced by $D$ is totally disconnected, and such that every cycle in $\Gamma$ contains at least two vertices in $D$. For a graph $\Gamma$ with this property, the full subgraph generated by the vertices in $V(\Gamma)-D$ is a forest; if each of the trees in this forest is collapsed to a point in $\Gamma$, the resulting graph is bipartite. Moreover, no vertex in $D$ is connected by an edge to more than one vertex in each tree of the forest $V(\Gamma)-D$. See Figure 1 for an example of a graph with the doubly breakable cycle property. In this example $D$ can be taken to be $D=\left\{d_{1}, d_{2}, d_{3}, d_{4}\right\}$.

Theorem B. Let $\Gamma$ be a graph. The right-angled Artin group $A \Gamma$ is poly-free of length 2 if and only if the graph $\Gamma$ has the doubly breakable cycle property. 


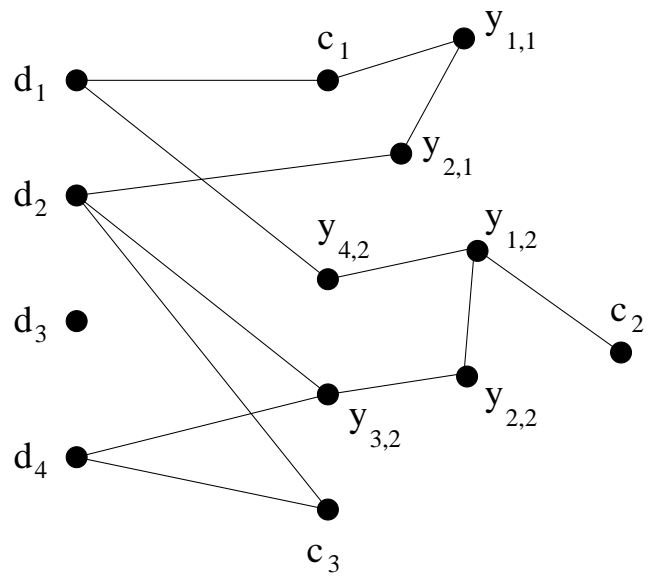

FigURE 1. An example of a graph with the doubly breakable cycle property

We note that Theorem $\mathrm{B}$ is valid in the context of both finite and infinite graphs.

Theorem $B$ can be used to show that each of the bounds in Theorem $\mathrm{A}$ are realized, as illustrated in the following two examples. First, consider the graph $C_{5}$ given by a 5 -cycle (i.e. a pentagon; see Figure 2). This graph has chromatic number $\operatorname{ch}\left(C_{5}\right)=3$, so Theorem $\mathrm{A}$ shows that the group $A C_{5}$ is poly-free with poly-free length at most 3. However, the pentagon satisfies the doubly breakable cycle property (for example, one can take $D=\{a, c\})$. Thus Theorem $\mathrm{B}$ improves this bound to $\operatorname{pfl}\left(A C_{5}\right) \leq 2$. Indeed, since $\operatorname{clq}\left(C_{5}\right)=2$, this group contains $\mathbb{Z}^{2}$ as a subgroup and is not free, so $\operatorname{pfl}\left(A C_{5}\right)=2$. Hence the lower bound on $\operatorname{pfl}(A \Gamma)$ given by the clique number in Theorem $\mathrm{A}$ is achieved in this example. Next, suppose that $P_{5}$ is a pentagonal prism (see Figure 2). In this case we have the same clique number and the same chromatic number as for the pentagon, i.e. clq $\left(P_{5}\right)=2$ and $\operatorname{chr}\left(P_{5}\right)=3$, but $P_{5}$ does not satisfy the doubly breakable cycle property. Indeed, in a graph that satisfies the doubly breakable cycle property exactly two non-neighboring vertices must be selected from each 4-cycle to be in the independent set of vertices $D$ breaking the cycles. Thus if we choose $a$ in $D$, then we must also have $b^{\prime}, c, d^{\prime}$ and $e$ in $D$ (the vertices indicated by squares in the diagram of $P_{5}$ in Figure 21). But $a$ and $e$ are neighbors, so they cannot both be in $D$. This shows that $a$ cannot be in $D$ and, by symmetry, no element can be in $D$. Since $D$ cannot be empty $P_{5}$ does not satisfy the doubly breakable cycle property. Theorems $\mathrm{A}$ and $\mathrm{B}$ show that $\operatorname{pfl}\left(A P_{5}\right) \leq 3$ and $\operatorname{pfl}\left(A P_{5}\right) \neq 2$. Thus $\operatorname{pfl}\left(A P_{5}\right)=3$ and the chromatic number upper bound is achieved for this second example.

If a graph $\Gamma$ satisfies the doubly breakable cycle property, the graph $\Gamma$ can be colored using three colors, one for the vertices in $D$, and two more for the vertices in $V(\Gamma)-D$ since their full subgraph is a forest. Consequently, 

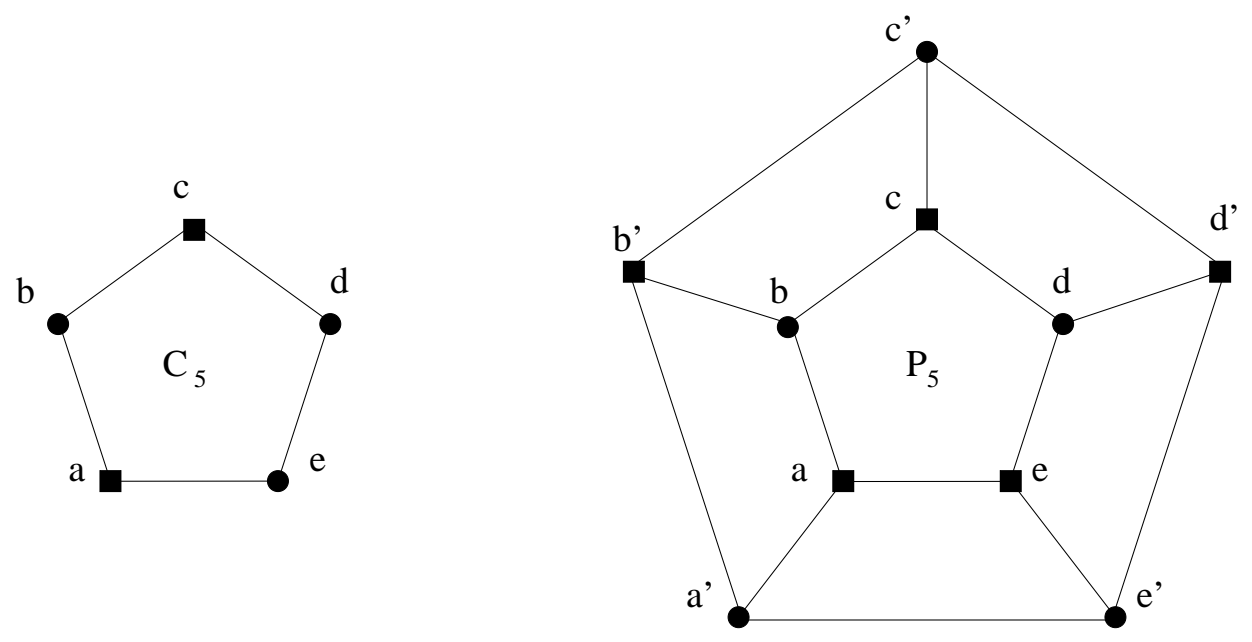

FiguRE 2. A pentagon and a pentagonal prism

Theorem $\mathrm{B}$ implies that whenever a right-angled Artin group $A \Gamma$ is poly-free of length 2 , then the defining graph $\Gamma$ must have chromatic number at most three.

Theorem C. A right-angled Artin group $A \Gamma$ is poly-fg-free of length 2 if and only if $\Gamma$ is a finite tree or a finite complete bipartite graph.

For the right-angled Artin group $A C_{5}$ discussed above, Theorem Cimplies that the group $A C_{5}$ is not poly-fg-free (of any length) even though it is polyfree of length 2. Indeed, by the results of D. Meier from [14] (see also [8]) the poly-fg-free length of a poly-fg-free group is equal to its rational homological dimension. Since the homological dimension of $A C_{5}$ is 2 (more on this later), $A C_{5}$ can only be poly-fg-free of length 2 . However, $C_{5}$ is neither a tree nor a complete bipartite graph and therefore $A C_{5}$ is not poly-fg-free.

Organization. Section 2 is a brief review of poly-free groups, right-angled Artin groups, and graph theoretic terminology. In Section 3 we prove one direction of Theorem $\mathrm{B}$, that every right-angled Artin group with poly-free length 2 has the doubly breakable cycle property, utilizing results of [16] and [3] on finiteness properties of subgroups of right-angled Artin groups. Section 4 contains the proof of Theorem C] utilizing a comparison of Euler characteristics for poly-free groups and right-angled Artin groups, together with the results of Section 3. In Section [5] an arbitrary right-angled Artin group is exhibited as a split extension of a free group by a right-angled Artin group on a subgraph, including an explicit description of the action, proving Theorem A Finally, in Section [ 6 we prove that for any graph with the doubly breakable cycle property, the corresponding right-angled Artin group $A \Gamma$ is a semidirect product of two free groups, using a refinement of 
the techniques of the previous section to construct the action. This result completes the proof of Theorem $\mathrm{B}$.

\section{BACKGROUND}

2.1. Groups. Throughout the text, $g^{a}$ denotes the conjugate $a^{-1} g a$.

Let $G=\langle S\rangle$ be a group generated by $S$. A word $w$ of length $k$ over $S \cup S^{-1}$ is a geodesic word if no word over $S \cup S^{-1}$ of length strictly less than $k$ represents the same element in $G$ as $w$ does. A total order defined on $S \cup S^{-1}$ induces a total order, called shortlex order, on all words over $S \cup S^{-1}$ in which shorter words always precede the longer ones and the words of the same length are ordered lexicographically according to the order defined on $S \cup S^{-1}$. A shortlex representative of an element $g \in G$ is the smallest word in the shortlex order that represents $g$. Such a representative is, by definition, geodesic.

2.2. Graphs. Throughout the paper, we assume that every graph is a simplicial graph; that is, a simple undirected graph. Therefore a graph $\Gamma$ is an ordered pair $\Gamma=(V, E)$ in which the set $V=V(\Gamma)$ is a set of vertices and $E=E(\Gamma)$ is a set of edges, which is a set of two element subsets of $V$. An edge $\{a, b\}$ has the vertices $a$ and $b$ as its endpoints. Two vertices $x$ and $y$ in $V$ are neighbors (are adjacent) if $\{x, y\}$ is an edge in $E$ (so no vertex is its own neighbor). A cycle in $\Gamma$ is a path of length at least 3 in which no vertex is repeated except for the initial and terminal one, which coincide. The clique number $\mathrm{clq}(\Gamma)$ of a graph $\Gamma$ is the largest size of a complete subgraph of $\Gamma$. Thus clq $(\Gamma)$ is the largest size of a subset $Q$ of $V$ for which every 2-element subset $\{a, b\} \subseteq Q$ is an edge in $E$. A proper coloring of a graph $\Gamma$ by $C$ is a labelling $\ell: C \rightarrow V$ of the vertices in $V$ by symbols from a set of colors $C$ in such a way that no two neighbors in $\Gamma$ are colored in the same color. Thus if $\{a, b\} \in E$ then $\ell(a) \neq \ell(b)$. The chromatic number of a graph $\Gamma$ is the smallest size of a set $C$ for which there exists a proper coloring of $\Gamma$ by $C$. A set of vertices $D$ is independent if it can be colored by the same color in some proper coloring of $\Gamma$. In other words, no two vertices in $D$ are adjacent.

2.3. Right-angled Artin groups. We freely use the following well known observation. If $\Gamma^{\prime}$ is a subgraph of $\Gamma$ induced by a set of vertices $X \subseteq V$, then the subgroup of $A \Gamma$ generated by the elements of $X$ is $A \Gamma^{\prime}$.

Throughout the text, given any homomorphism $\phi: A \Gamma \rightarrow F_{q}$ from a rightangled Artin group to a free group, the set $D:=\{v \in V(\Gamma) \mid \phi(v)=1\}$ is called the set of dead vertices, the set $L:=V(\Gamma)-D$ is the set of living vertices, and the full subgraph $\Gamma_{L}$ generated by $L$ is the living subgraph of $\Gamma$, with respect to $\phi$.

Lemma 2.1. 9 Every geodesic representative of an element $t \in A \Gamma$ can be obtained from any other representative of $t$ by finite number of applications of the following operations: 
(1) Eliminate a subword of the form $x x^{-1}$ or $x^{-1} x$ with $x \in V(\Gamma)$.

(2) If $x, y \in V(\Gamma)$ are adjacent in $\Gamma$, replace a single occurrence of $x^{ \pm} y^{ \pm}$ by $y^{ \pm} x^{ \pm}$.

In particular, every geodesic representative of an element $t \in A \Gamma_{L}$ can be obtained from any other geodesic representative of $t$ by finite number of applications of operation 2.

2.4. Poly-free groups. Throughout the text, when $G$ is a semidirect product of two free groups, we will write $G=F_{k} \rtimes F_{q}$ with associated canonical homomorphism $\phi: G \rightarrow F_{q}$, so that the rank of the $\operatorname{kernel} \operatorname{ker}(\phi)$ is $k$ and the rank of the associate quotient is $q$.

Proposition 2.2. If $G$ is poly-free with length $N$ and $H \leq G$, then $H$ is poly-free with length $\leq N$.

Proof. Given a poly-free tower $1=G_{0} \unlhd G_{1} \unlhd \cdots \unlhd G_{N}=G$ for $G$, then the tower $1=G_{0} \cap H \unlhd G_{1} \cap H \unlhd \cdots \unlhd G_{N} \cap H=H$ is a poly-free tower for $H$.

Proposition 2.3. If $G$ has a normal free subgroup $H$ and the quotient $G / H$ is poly-free with $\operatorname{pfl}(G / H)=N$, then $G$ is poly-free with $\operatorname{pfl}(G) \leq N+1$.

Proof. Let $\phi: G \rightarrow G / H$ be the canonical homomorphism. Given a polyfree tower $1=Q_{0} \unlhd Q_{1} \unlhd \cdots \unlhd Q_{N}=G / H$ for $G / H$, then the tower $1 \unlhd \phi^{-1}\left(Q_{0}\right)=H \unlhd \phi^{-1}\left(Q_{1}\right) \unlhd \cdots \unlhd \phi^{-1}\left(Q_{N}\right)=G$ is a poly-free tower for $G$.

\section{Poly-freeness of length 2 implies the doubly Breakable CYCLE PROPERTY}

Before proving the statement of the title of this section in Proposition 3.5 we begin with a few lemmas.

Lemma 3.1. Let $\Gamma$ be a graph with $A \Gamma=F_{k} \rtimes F_{q}$ for free groups $F_{k}$ and $F_{q}$ of finite or infinite rank. Let $\phi: A \Gamma \rightarrow F_{q}$ be the canonical homomorphism and let $\Gamma_{L}$ be the corresponding living subgraph. If $\Gamma_{\alpha}$ is a connected subgraph of $\Gamma_{L}$, then the subgroup $\left\langle\left\{\phi(u) \mid u \in \Gamma_{\alpha}\right\}\right\rangle$ of $F_{q}$ is isomorphic to $\mathbb{Z}$.

Proof. Fix a connected subgraph $\Gamma_{\alpha}$ in $\Gamma_{L}$. The images $\phi(u), u \in \Gamma_{\alpha}$ are nontrivial elements in the free group $F_{q}$. Using the fact that $\Gamma_{\alpha}$ is connected and [13. Prop. I.2.18] that says that the commuting relation is an equivalence relation on the set of nontrivial elements in a free group, we conclude that the group $\left\langle\left\{\phi(u) \mid u \in \Gamma_{\alpha}\right\}\right\rangle$ is abelian. However, the only abelian subgroup of $F_{q}$ is $\mathbb{Z}$ and the conclusion follows.

Lemma 3.2. Let $\Gamma$ be a graph with $A \Gamma=F_{k} \rtimes F_{q}$ for free groups $F_{k}$ and $F_{q}$ of finite or infinite rank. Let $\phi: A \Gamma \rightarrow F_{q}$ be the canonical homomorphism and let $D:=\{v \in V(\Gamma) \mid \phi(v)=1\}$ be the set of dead vertices. If $d \in D$ and $a_{1}, \ldots, a_{n} \in L=V(\Gamma)-D$ are all adjacent to $d$, then the subgroup $\left\langle\phi\left(a_{1}\right), \ldots, \phi\left(a_{n}\right)\right\rangle$ of $F_{q}$ is free of rank $n$. 
Proof. Suppose that there exists a nontrivial word $u_{1} \cdots u_{m}$ with each $u_{i} \in$ $\left\{a_{1}, \ldots, a_{n}\right\}^{ \pm 1}$ and $\phi\left(u_{1}\right) \cdots \phi\left(u_{m}\right)=1$. Then $u_{1} \cdots u_{m} \in \operatorname{ker}(\phi)$, and since each $a_{i}$ is adjacent to $d$, the subgroup $\left\langle d, u_{1} \cdots u_{m}\right\rangle$ of $\operatorname{ker}(\phi)$ is isomorphic to $\mathbb{Z}^{2}$. This contradicts the hypothesis that $\operatorname{ker}(\phi)=F_{k}$ is free.

Lemma 3.3. Let $\Gamma$ be a finite graph, let $\phi: A \Gamma \rightarrow Z$ be any group homomorphism from $A \Gamma$ to an infinite cyclic group $Z=\langle z\rangle$, and let $\rho: A \Gamma \rightarrow Z$ be the homomorphism defined by $\rho(v):=z$ for a vertex $v \in V(\Gamma)$ if $\phi(v) \neq 1$ and $\rho(v):=1$ if $\phi(v)=1$. Then $\operatorname{ker}(\phi)$ is free if and only if $\operatorname{ker}(\rho)$ is free.

Proof. Let $D:=\{w \in V(\Gamma) \mid \phi(w)=1\}$ and for each $v \in V(\Gamma)-D$, let $n_{v} \in \mathbb{Z}$ be the unique integer such that $z^{n_{v}}=\phi(v)$ in $Z$.

First suppose that the group $\operatorname{ker}(\rho)$ is not free. Let $N$ be the least common multiple of the numbers $\left|n_{v}\right|$ for $v \in V(\Gamma)-D$. Then for each $v \in V(\Gamma)-D$, there exists an integer $a_{v}$ such that $n_{v} a_{v}=N$. Define $a_{w}:=1$ for each $w \in D$. Let $\mu: Z \rightarrow Z$ be the homomorphism $z^{k} \mapsto z^{N k}$ given by taking the $N$-th power in $Z$. The composition $\mu \circ \rho: A \Gamma \rightarrow Z$ has kernel $\operatorname{ker}(\mu \circ \rho)=$ $\operatorname{ker}(\rho)$. Define $\Theta: A \Gamma \rightarrow A \Gamma$ by $\Theta(v):=v^{a_{v}}$ for all $v \in V(\Gamma)$; this defines a homomorphism of groups. Moreover, the compositions $\phi \circ \Theta=\mu \circ \rho$. Hence $\operatorname{ker}(\rho)=\operatorname{ker}(\mu \circ \rho)=\operatorname{ker}(\phi \circ \Theta)$.

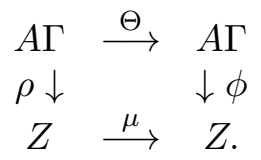

Suppose that $1 \neq g \in \operatorname{ker}(\Theta)$. Put a total ordering on $V(\Gamma)$, and let $g={ }_{A \Gamma}$ $v_{1}^{j_{1}} v_{2}^{j_{2}} \cdots v_{k}^{j_{k}}$ be the shortlex least representative of $g$ with each $v_{i} \in V(\Gamma)$, $j_{i} \in \mathbb{Z}$, and $v_{i} \neq v_{i+1}$. Then $\Theta(g)={ }_{A \Gamma} v_{1}^{j_{1} a_{v_{1}}} v_{2}^{j_{2} a_{v_{2}}} \cdots v_{k}^{j_{k} a_{v_{k}}}={ }_{A \Gamma} 1$. Since $g \neq 1$, the word $v_{1}^{j_{1}} v_{2}^{j_{2}} \cdots v_{k}^{j_{k}}$ is not empty, so there must be commutation relations such that for some indices $i<m$ we have $v_{i}=v_{m}, j_{i} j_{m}<0$, and $v_{i}$ commutes with $v_{n}$ for all $i<n<m$, so that cancellation occurs between $v_{i}^{j_{i} a_{v_{i}}}$ and $v_{m}^{j_{m} a_{v_{m}}}$. However, in that case cancellation must also be possible in the normal form word $v_{1}^{j_{1}} v_{2}^{j_{2}} \cdots v_{k}^{j_{k}}$, giving a contradiction. Therefore $\operatorname{ker}(\Theta)=1$.

We now have that $\Theta$ is a monomorphism, and $\Theta(\operatorname{ker}(\rho))=\Theta(\operatorname{ker}(\mu \circ \rho))=$ $\Theta(\operatorname{ker}(\phi \circ \Theta)) \leq \operatorname{ker}(\phi)$. Hence $\operatorname{ker}(\rho)$ is isomorphic to a subgroup of $\operatorname{ker}(\phi)$. Using the Nielsen-Schreier Subgroup Theorem, that every subgroup of a free group is free, together with the hypothesis that $\operatorname{ker}(\rho)$ is not free, we have that $\operatorname{ker}(\phi)$ cannot be a free group.

Next suppose that $\operatorname{ker}(\phi)$ is not free. Define the function $\Psi: A \Gamma \rightarrow A \Gamma$ by $\Psi(v):=v^{n_{v}}$ for $v \in V(\Gamma)-D$ and $\Psi(w):=w$ for $w \in D$. Then $\phi=\rho \circ \Psi$. An argument similar to the proof above for $\Theta$ shows that $\Psi$ is a monomorphism of groups, so $\Psi$ restricts to an isomorphism from $\operatorname{ker}(\phi)$ to a subgroup of $\operatorname{ker}(\rho)$. Since $\operatorname{ker}(\phi)$ is not free, then $\operatorname{ker}(\rho)$ also cannot be free.

Note that Lemma 3.3 is the only lemma in this section for which the graph must be finite. In the proof of Theorem B, this lemma is applied only 
to a cycle $\Gamma^{\prime}$ in $\Gamma$, which is finite, and hence Theorem B is valid for both finite and infinite graphs $\Gamma$.

Lemma 3.4. The group $\mathbb{Z}^{n}$ has poly-free length equal to $n$.

Proof. Since $\mathbb{Z}^{n}=\underbrace{\mathbb{Z} \times \mathbb{Z} \times \cdots \times \mathbb{Z}}_{n}$ is an iterated direct product of $n$ free groups, $\mathbb{Z}^{n}$ is poly-free with length at most $n$.

To show that $\mathbb{Z}^{n}$ cannot have poly-free length less than $n$, assume that $\mathbb{Z}^{n}=\left(\ldots\left(F_{n_{1}} \rtimes F_{n_{2}}\right) \rtimes \ldots\right) \rtimes F_{n_{k}}$, for some (finite or infinite) $n_{i}, i=1, \ldots, k$. Since $\mathbb{Z}^{n}$ is abelian, we must have $n_{1}=n_{2}=\cdots=n_{k}=1$. Thus $\mathbb{Z}^{n}$ can be generated by $k$ elements (one for each $F_{n_{i}}$ ). However, $Z^{n}$ cannot be generated by fewer than $n$ elements, which shows that $n \leq k$.

Note that Lemma 3.4 shows that the right-angled Artin group whose graph $\Gamma$ is a triangle cannot be poly-free of length 2 .

Proposition 3.5. If $\Gamma$ is a graph and the right-angled Artin group $A \Gamma$ is poly-free of length 2 , then the graph $\Gamma$ has the doubly breakable cycle property.

Proof. The group $A \Gamma$ can be written as a semidirect product $A \Gamma=F_{k} \rtimes F_{q}$ where $F_{k}$ and $F_{q}$ are free groups of ranks $k$ and $q$, respectively, and $k, q \in$ $\mathbb{N} \cup\{\infty\}$. Let $\phi: A \Gamma \rightarrow F_{q}$ be the canonical surjection, with $\operatorname{ker}(\phi)=F_{k}$. Let $D$ be the set of vertices $v$ in $\Gamma$ with $\phi(v)=1$; that is, $v \in \operatorname{ker}(\phi)$.

If $d_{1}$ and $d_{2}$ are vertices in $D$, then the subgroup $\left\langle d_{1}, d_{2}\right\rangle<A \Gamma$ generated by $d_{1}$ and $d_{2}$ must also be contained in $\operatorname{ker}(\phi)$. Since $\operatorname{ker}(\phi)$ is free, the subgroup $\left\langle d_{1}, d_{2}\right\rangle$ of $\operatorname{ker}(\phi)$ is free. Hence $d_{1}$ and $d_{2}$ cannot be joined by an edge in $\Gamma$. Thus the subgraph induced by $D$ is totally disconnected, i.e., $D$ is an independent set of vertices in $\Gamma$.

Suppose that $\Gamma^{\prime}$ is a cycle in the graph $\Gamma$. Then $\Gamma^{\prime}$ contains at least 3 vertices. The subgroup $A \Gamma^{\prime}$ of $A \Gamma$ is again a right-angled Artin group, and Proposition 2.2 says that $A \Gamma^{\prime}$ is poly-free with length at most 2. Lemma 3.4 says that $\mathbb{Z}^{3}$ is not poly-free with length less than 3 , so the cycle $\Gamma^{\prime}$ cannot have length 3 . Thus $\Gamma^{\prime}$ contains at least 4 vertices.

If $V\left(\Gamma^{\prime}\right) \cap D$ contains less than two vertices, then the full subgraph of $\Gamma^{\prime}$ generated by the vertices in $V\left(\Gamma^{\prime}\right)-D$ is connected. In this case Lemma 3.1 shows that the restriction $\left.\phi\right|_{A \Gamma^{\prime}}: A \Gamma^{\prime} \rightarrow F_{q}$ has range $\langle f\rangle=\mathbb{Z}$ for some $f \in F_{q}$. Since $\operatorname{ker}\left(\left.\phi\right|_{A \Gamma^{\prime}}\right)<\operatorname{ker}(\phi)$, then $\operatorname{ker}\left(\left.\phi\right|_{A \Gamma^{\prime}}\right)$ is also a free group.

If $V\left(\Gamma^{\prime}\right) \cap D=\emptyset$, then $\phi(v) \neq f^{0}$ for all $v \in V\left(\Gamma^{\prime}\right)$. Define the function $\rho: A \Gamma^{\prime} \rightarrow \mathbb{Z}$ by $\rho(v)=f$ for all $v \in V\left(\Gamma^{\prime}\right)$. Then Lemma 3.3 says that $\operatorname{ker}(\rho)$ is free. Since the graph $\Gamma^{\prime}$ is connected, 16. Theorem 6.3] shows that $\operatorname{ker}(\rho)$ is finitely generated. The cycle $\Gamma^{\prime}$ is a flag complex, since $\Gamma^{\prime}$ is not a triangle, and this flag complex is not simply connected. The Main Theorem of [3] shows that $\operatorname{ker}(\rho)$ is not finitely presented. Since a free group cannot be finitely generated but not finitely presented, we have a contradiction.

If there is exactly one vertex $d$ in $V\left(\Gamma^{\prime}\right) \cap D$, then the images $\phi(a)$ and $\phi(b)$ of the two neighbors $a$ and $b$ of $d$ in the cycle $\Gamma^{\prime}$ must generate a free group of rank 2 in $F_{q}$ (by Lemma 3.2). On the other hand, we already 
established that the range of $\left.\phi\right|_{A \Gamma^{\prime}}: A \Gamma^{\prime} \rightarrow F_{q}$ is cyclic, resulting again in a contradiction.

\section{Poly-FG-Freeness of Length 2}

In this section we prove Theorem $\mathrm{C}$ using an analysis of Euler characteristics of right-angled Artin groups and poly-free groups.

Lemma 4.1. Let $\Gamma$ be a finite graph with $A \Gamma=F_{k} \rtimes F_{q}$ for free groups $F_{k}$ and $F_{q}$ of finite rank. Let $\phi: A \Gamma \rightarrow F_{q}$ be the canonical homomorphism, let $D:=\{v \in V(\Gamma) \mid \phi(v)=1\}$ be the set of dead vertices, and for each $d \in D$, let $N_{d}$ denote the set of vertices adjacent to $d$ in $\Gamma$. Then the image of the subgroup generated by $N_{d}$ under the map $\phi$ has finite index in $F_{q}$, and $k \geq \sum_{d \in D}\left[F_{q}: \phi\left(\left\langle N_{d}\right\rangle\right)\right]$.

Proof. We begin by finding a presentation for the subgroup $K:=\operatorname{ker}(\phi)=$ $F_{k}$ of $A \Gamma$ using the Reidemeister-Schreier procedure, following the notation in [13. Proposition II.4.1]. Let $F(V(\Gamma))$ be the free group on the vertices of $\Gamma$, let $\alpha: F(V(\Gamma)) \rightarrow A \Gamma$ be the canonical epimorphism, and let $\widetilde{K}:=\alpha^{-1}(K)$. Then $F(V(\Gamma)) / \widetilde{K} \cong F_{q}$. Choose a Schreier transversal $T$ for $\widetilde{K}$ in $F(V(\Gamma))$.

For any element $y \in F(V(\Gamma))$, let $\bar{y}$ denote the element of $T$ for which $K y=K \bar{y}$. For $t \in T$ and $v \in V(\Gamma)$, define $\gamma(t, v):=t v \overline{t v}^{-1}$. Given $d \in D$ and $t \in T$, then $\overline{t d}=t$. The element $d_{t}:=t d t^{-1}=\gamma(t, d)$ is a conjugate of a nontrivial element in $A \Gamma$, so $d_{t}$ itself is not trivial. Given $a \in L$ and $t \in T$, define $a_{t}:=t a \overline{t a}^{-1}=\gamma(t, a)$ as well. The subset $S$ of nontrivial elements in the set

generates $K$.

$$
\left\{d_{t} \mid t \in T, d \in D\right\} \cup\left\{a_{t} \mid t \in T, a \in L\right\}
$$

For $t \in T$ and $v \in V(\Gamma)$, also define $\gamma\left(t, v^{-1}\right):=t v^{-1}\left(\overline{t v^{-1}}\right)^{-1}$. If $t \in T$, $d \in D$, and $a \in L$, then $\gamma\left(t, d^{-1}\right)=d_{t}^{-1}$ and $\gamma\left(t, a^{-1}\right)=\left(a_{\overline{t a^{-1}}}\right)^{-1}$. Given any word $v=v_{1} \cdots v_{m}$ with each $v_{i} \in V(\Gamma)^{ \pm 1}$, define

$$
\tau(v):=\gamma\left(1, v_{1}\right) \gamma\left(\overline{v_{1}}, v_{2}\right) \cdots \gamma\left(\overline{v_{1} \cdots v_{m-1}}, v_{m}\right) .
$$

Note that for each $t \in T$ and each relator $r=[u, v] \in R$, the element $\tau(t)$ freely reduces to 1 , and

$$
\tau\left(t r t^{-1}\right)=\gamma(t, u) \gamma(\overline{t u}, v) \gamma\left(\overline{t u v}, u^{-1}\right) \gamma\left(\overline{t u v u^{-1}}, v^{-1}\right) .
$$

The latter words form the defining relators of the presentation for $K=$ $\operatorname{ker}(\phi)$. In particular, a defining set of relations is given by

$$
\begin{aligned}
R:= & \left\{d_{t} a_{t}=a_{t} d_{\overline{t a}} \mid t \in T, d \in D, a \in L,\{d, a\} \in E(\Gamma)\right\} \cup \\
& \left\{a_{t} b_{\overline{t a}}=b_{t} a_{\overline{t b}} \mid t \in T, a, b \in L,\{a, b\} \in E(\Gamma)\right\}
\end{aligned}
$$

and the group $K$ is presented by $\langle S \mid R\rangle$.

Abelianizing this presentation yields a presentation for $K_{a b}=\mathbb{Z}^{k}$. The subgroup $H$ of $K_{a b}$ generated by the elements of

$$
D_{T}:=\left\{d_{t} \mid d \in D, t \in T\right\}
$$


is a free abelian direct factor of $K_{a b}$ presented by

$$
H=\left\langle D_{T} \mid\left\{d_{t}=d_{\overline{t a}} \mid t \in T, d \in D, a \in N_{d}\right\}\right\rangle_{a b} .
$$

Since all relations in this presentation are equalities between generators, the rank of $H$ is the number of equivalence classes of generators. Note that two generators $d_{t}$ and $d_{s}$ are equal in $H$ if and only if there exists a sequence of relations $d_{t}=d_{\overline{t a_{1}}}=d_{\overline{t a_{1} a_{2}}}=\cdots=d_{\overline{t a_{1} \cdots a_{m}}}=d_{s}$ with $s=\overline{t a_{1} \cdots a_{m+1}}$ and each $a_{i} \in N_{d}^{ \pm 1}$. This holds if and only if $K t a_{1} \cdots a_{m+1}=K s$, which is satisfied if and only if $\phi(t) \phi\left(a_{1}\right) \cdots \phi\left(a_{m+1}\right)=\phi(s)$. Then $d_{t}$ and $d_{s}$ are equal in $H$ if and only if $\phi(t)$ and $\phi(s)$ are in the same coset of $\phi\left(\left\langle N_{d}\right\rangle\right)$ in $F_{q}$. Therefore the rank of $H$ is equal to the sum of indices $\sum_{d \in D}\left[F_{q}: \phi\left(\left\langle N_{d}\right\rangle\right)\right]$. Since the rank of $K_{a b}$ is $k$ we have

$$
k \geq \operatorname{rank}(H)=\sum_{d \in D}\left[F_{q}: \phi\left(\left\langle N_{d}\right\rangle\right)\right] .
$$

In particular, the index $\left[F_{q}: \phi\left(\left\langle N_{d}\right\rangle\right)\right]$ is finite for all $d \in D$.

Theorem C. A right-angled Artin group AГ is poly-fg-free of length 2 if and only if $\Gamma$ is either a finite tree or a finite complete bipartite graph.

Proof. If $\Gamma$ is the complete bipartite graph $K_{k, q}$, then $A \Gamma$ is the direct product $F_{k} \times F_{q}$ of free groups of ranks $k$ and $q$. On the other hand, if $\Gamma$ is a tree on $n$ vertices, the Artin group $A \Gamma$ is a semidirect product $F_{n-1} \rtimes \mathbb{Z}[11$, Proposition 4.6].

Conversely, assume for the rest of this proof that $A \Gamma$ is a poly-fg-free group of length 2 . Since $A \Gamma$ is finitely generated, the graph $\Gamma$ must be finite (since the abelianization of $A \Gamma$ is $\mathbb{Z}^{V}$, any generating set of $A \Gamma$ has at least $|V|$ elements).

There exists a split short exact sequence

$$
1 \rightarrow F_{k} \rightarrow A \Gamma \stackrel{\phi}{\rightarrow} F_{q} \rightarrow 1,
$$

such that the ranks $k$ and $q$ of the free groups are finite and positive. By Proposition 3.5 the graph $\Gamma$ must satisfy the doubly breakable cycle property. Moreover, the proof of that proposition shows that the set of dead vertices $D:=\{v \in V(\Gamma) \mid \phi(v)=1\}$ associated to $\phi$ is an independent set of vertices in $\Gamma$ such that every cycle in $\Gamma$ meets $D$ at least twice. Since $A \Gamma$ is not poly-fg-free of length 1 , the living subgraph $\Gamma_{L}$ is not empty.

Since semidirect products of free groups are torsion free, the Euler characteristic of the semidirect product $A \Gamma=F_{k} \rtimes F_{q}$ is given by $\chi(A \Gamma)=$ $\chi\left(F_{k}\right) \chi\left(F_{q}\right)$ (see [5. Proposition IX.7.3(d)]). Therefore, given that the Euler characteristic of a free group $F_{r}$ of rank $r$ is $\chi\left(F_{r}\right)=1-r$,

$$
\chi(A \Gamma)=(k-1)(q-1) .
$$

The Euler characteristic of a right-angled Artin group can be computed using a $K(A \Gamma, 1)$ space. The doubly breakable cycle property implies that $\Gamma$ contains edges but does not contain any triangles. In this case the standard 
2-complex associated to the standard presentation (from Section 10 of $A \Gamma$ is a $K(A \Gamma, 1)$ [16, Theorem 7.3], and the associated chain complex is

$$
\cdots 0 \rightarrow \bigoplus_{|E(\Gamma)|} \mathbb{Z}^{\partial_{2}} \rightarrow \bigoplus_{|V(\Gamma)|} \mathbb{Z}^{\partial_{1}} \mathbb{Z} \rightarrow 0
$$

(this complex can also be obtained as the co-invariants of the free $\mathbb{Z} A \Gamma$ module resolution in [6]). A straightforward computation shows that all of the boundary maps $\partial_{i}$ in this complex are trivial, so $H_{0}(A \Gamma)=\mathbb{Z}, H_{1}(A \Gamma)=$ $\mathbb{Z}^{v}$, and $H_{2}(A \Gamma)=Z^{e}$ where $v:=|V(\Gamma)|$ and $e:=|E(\Gamma)|$. Thus the Euler characteristic of $A \Gamma$ is also

$$
\chi(A \Gamma)=1-v+e .
$$

Denote the connected components of the nonempty graph $\Gamma_{L}$ by $C_{1}, \ldots, C_{c}$. Each of these components is a tree with $n_{j}>0$ vertices, and hence $n_{j}-1$ edges, for $1 \leq j \leq c$. Let $\delta:=|D|$ and denote the degrees of the vertices $d_{1}, \ldots d_{\delta}$ in $D$ by $g_{1}, \ldots, g_{\delta}$. Rewriting Equation (2) yields

$$
\begin{aligned}
\chi(A \Gamma)=1-\left(\delta+\sum_{j=1}^{c} n_{j}\right) & +\left(\sum_{j=1}^{c}\left(n_{j}-1\right)+\sum_{i=1}^{\delta} g_{i}\right)= \\
& =1-\delta-c+\sum_{i=1}^{\delta} g_{i}=1-c+\sum_{i=1}^{\delta}\left(g_{i}-1\right) .
\end{aligned}
$$

For each $1 \leq i \leq \delta$, let $N_{i}$ denote the set of vertices adjacent to $d_{i}$. Lemma 3.2 says that the subgroup $\phi\left(\left\langle N_{i}\right\rangle\right)$ of $F_{q}$ is free of rank equal to the degree $g_{i}$ of $d_{i}$. Using the Schreier Formula, $\left(\operatorname{rank}\left(F_{q}\right)-1\right)\left[F_{q}: \phi\left(\left\langle N_{i}\right\rangle\right)\right]=$ $\operatorname{rank}\left(\phi\left(\left\langle N_{i}\right\rangle\right)-1\right)$, so

$$
g_{i}-1=(q-1)\left[F_{q}: \phi\left(\left\langle N_{i}\right\rangle\right)\right] .
$$

According to Lemma 4.1. $\sum_{i=1}^{\delta}\left[F_{q}: \phi\left(\left\langle N_{d_{i}}\right\rangle\right)\right] \leq k$. Thus, taking into account the non-negativity of $q-1$,

$$
\sum_{i=1}^{\delta}\left(g_{i}-1\right)=(q-1) \sum_{i=1}^{\delta}\left[F_{q}: \phi\left(\left\langle N_{d_{i}}\right\rangle\right)\right] \leq k(q-1) .
$$

Combining this with Equation (10) and Equation (3), then

$$
(k-1)(q-1) \stackrel{\text { 雨 }}{=} \chi(\Gamma) \stackrel{\text { 芆 }}{=} 1-c+\sum_{i=1}^{\delta}\left(g_{i}-1\right) \leq 1-c+k(q-1),
$$

which implies that $c \leq q$.

Lemma 3.1 says that for each component $C_{j}$, there exists an element $f_{j}$ in $F_{q}$ such that all vertices from the component $C_{j}$ are mapped by $\phi$ to a power of $f_{j}$. Since $\phi$ is onto and the dead vertices in $D$ are mapped to the 
identity in $F_{q}, f_{1}, \ldots, f_{c}$ generate $F_{q}$, which implies that $q \leq c$. Using the inequality at the end of the previous paragraph, then

$$
q=c .
$$

If $q=c=1$, then the living subgraph $\Gamma_{L}$ of $\Gamma$ is a single tree. Since the kernel $\operatorname{ker}(\phi)=F_{k}$ is finitely generated, [16. Theorem 6.1] says that every dead vertex in $D$ must be adjacent to a vertex in $\Gamma_{L}$. The doubly breakable cycle property says that there cannot be a cycle in $\Gamma$ that meets a dead vertex in $D$ only once, which implies that for each $d \in D, d$ cannot be the endpoint of two different edges whose other endpoints lie in $\Gamma_{L}$. Therefore in this case the graph $\Gamma$ is also a tree.

Finally suppose that $q=c \geq 2$. As in the previous paragraph, the doubly breakable cycle property says that for each $d_{i} \in D, d_{i}$ cannot be the endpoint of two different edges whose other endpoints lie in the same component $C_{j}$ of $\Gamma_{L}$. Hence each degree $g_{i} \leq c$, so $\sum_{i=1}^{\delta} g_{i} \leq c \delta$. Using Equation (4), Equation (11), and Equation (3), then

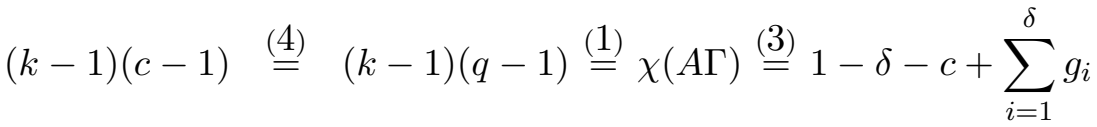

$$
\begin{aligned}
& \leq 1-\delta-c+c \delta=(c-1)(\delta-1) .
\end{aligned}
$$

Since $c \geq 2$, therefore

$$
k \leq \delta .
$$

Note that equality holds if and only if each vertex in $D$ has degree $c$; i.e., there exists a single edge between each vertex in $D$ and each component of $\Gamma_{L}$.

Since $A \Gamma=F_{k} \rtimes F_{q}$, the group $A \Gamma$ can be generated by $k+q$ elements. However, the minimal number of generators for the right-angled Artin group $A \Gamma$ is the number $v=\delta+\sum_{j=1}^{c} n_{j}$ of vertices in $\Gamma$, which in turn is at least as large as $\delta+c$. Thus

$$
\delta+c \leq k+q
$$

Note that equality in this case is possible only if $\delta+c=v=\delta+\sum_{j=1}^{c} n_{j}=$ $k+q$, and so $c=\sum_{j=1}^{c} n_{j}$. Thus equality implies that each $n_{j}=1$; i.e., each component $C_{j}$ of $\Gamma_{L}$ is a single vertex.

Using the fact that $q=c$, Inequality (15) and Inequality (6) imply that $\delta=k$, so equality holds both in (5) and in (6). Therefore $D$ and $L$ are each independent sets of vertices in $\Gamma$, and there is an edge between each vertex in $D$ and each vertex in $L$. Thus in this case $\Gamma$ is a complete bipartite graph.

\section{Every Right-ANGLED Artin Group IS POLY-FREE}

Given a graph $\Gamma$ with finite chromatic number greater than one, let $D$ be the set of vertices in one of the colors and let $L:=V(\Gamma)-D$ be the vertices 
in the other colors. Let $\Gamma_{L}$ be the full subgraph of $\Gamma$ induced by $L$. If we define a homomorphism $\phi: A \Gamma \rightarrow A \Gamma_{L}$ by $\phi(d)=1$ for $d \in D$ and $\phi(a)=a$ for $a \in L$, then $D$ is the set of dead vertices and $\Gamma_{L}$ is the living subgraph associated to this homomorphism. In the following proof we construct a free group $F$ (isomorphic to $\operatorname{ker}(\phi)$ ) and an action of $A \Gamma_{L}$ on $F$, and exhibit directly that $A \Gamma$ is isomorphic to the semidirect product $F \rtimes A \Gamma_{L}$ of a free group with $A \Gamma_{L}$.

Theorem \$, Let $\Gamma$ be a finite graph or, more generally, a graph of finite chromatic number $\operatorname{chr}(\Gamma)$ and finite clique number clq $(\Gamma)$. The right-angled Artin group $A \Gamma$ is poly-free. Moreover,

$$
\operatorname{clq}(\Gamma) \leq \operatorname{pfl}(A \Gamma) \leq \operatorname{chr}(\Gamma),
$$

and there exists a poly-free tower for $A \Gamma$ of length $\operatorname{chr}(\Gamma)$.

Proof. To prove poly-freeness and the upper bound on the poly-free length, we induct on $\operatorname{chr}(\Gamma)$. If $\operatorname{chr}(\Gamma)=1$, then $\Gamma$ is totally disconnected, so $A \Gamma$ is free, and hence poly-free of length 1 . Next suppose that $\operatorname{chr}(\Gamma) \geq 2$, and that for every graph $\Gamma^{\prime}$ with $\operatorname{chr}\left(\Gamma^{\prime}\right)<\operatorname{chr}(\Gamma)$, the group $A \Gamma^{\prime}$ is poly-free and has a poly-free tower of length $\operatorname{chr}\left(\Gamma^{\prime}\right)$.

Choose a coloring of $\Gamma$ in $\operatorname{chr}(\Gamma)$ colors, one of which is gray. Let $D$ be the set of vertices in $V=V(\Gamma)$ colored in gray, $L=V-D$ be the set of vertices colored in a different color, $\Gamma_{L}$ be the subgraph of $\Gamma$ induced by $L$ and $A \Gamma_{L}$ be the corresponding right-angled Artin group. Then $\operatorname{chr}\left(\Gamma_{L}\right)=\operatorname{chr}(\Gamma)-1$ and the inductive assumption implies that there exists a poly-free tower for $A \Gamma_{L}$ of length $\operatorname{chr}(\Gamma)-1$.

In the discussion that follows, a geodesic representative of an element $t \in$ $A \Gamma_{L}$ means a geodesic word in the alphabet $L^{ \pm 1}$. For any vertex $v \in V(\Gamma)$, denote by $N_{v}$ the set of vertices adjacent to $v$; i.e., the neighbors of $v$. For each $d \in D$, define a set of symbols

$$
T_{d}:=\left\{d_{t} \mid t \in A \Gamma_{L} \text {, no geodesic rep. of } t \text { starts with a letter in } N_{d}^{ \pm 1}\right\} \text {. }
$$

Let $F\left(T_{d}\right)$ be the free group over $T_{d}$ and let $F$ be the free group

$$
F:=*_{d \in D} F\left(T_{d}\right) .
$$

For each generator $a \in L$, define an endomorphism $\alpha_{a}: F \rightarrow F$ by

$$
\alpha_{a}\left(d_{t}\right):=\left\{\begin{array}{ll}
d_{t a}, & d_{t a} \in T_{d} \\
d_{t}, & d_{t a} \notin T_{d}
\end{array},\right.
$$

for all $d \in D$ and $d_{t} \in T_{d}$. Since $F$ is a free group, this definition of $\alpha_{a}$ on the generators of $F$ extends to an endomorphism on $F$. In order to show that $a \mapsto \alpha_{a}$ extends to an action of $A \Gamma_{L}$ on $F$, we first need to consider when the conditions $d_{t} \in T_{d}$ and $d_{t a} \notin T_{d}$ occur simultaneously.

Assume that $d_{t} \in T_{d}$. If $d_{t a} \notin T_{d}$, then there exists a geodesic representative $w$ of $t a$ that begins with a letter in $N_{d}^{ \pm 1}$. Consider the word $w a^{-1}$ representing $t$. Since $d_{t} \in T_{d}$ the word $w a^{-1}$ cannot be geodesic. By 
Lemma 2.1 we can write $w$ as $u_{1} a u_{2}$ where $a$ commutes with all the letters in $u_{2}$. The word $u_{1} u_{2}$ is a geodesic representative of $t$. As such, it cannot start in $N_{d}^{ \pm 1}$. Thus the geodesic word $w=u_{1} a u_{2}$ representing $t a$ and the geodesic word $u_{1} u_{2}$ representing $t$ start in a different letter. This is possible only when $u_{1}$ is empty. Thus $w=a u_{2}$ is a geodesic representative of $t a$ that starts in $N_{d}^{ \pm 1}$ and $a$ commutes with all letters in $u_{2}$, i.e., $a$ commutes with $d$ and all the letters in $u_{2}$. However, $u_{2}$ is a geodesic representative of $t$. Since any other geodesic representative of $t$ can be obtained from $u_{2}$ by commuting letters we conclude that $a$ commutes with $d$ and all the letters in any geodesic representative of $t$. A similar proof shows that, conversely, if $a$ commutes with $d$ and all letters in any geodesic representative of $t$ then $d_{t a}$ cannot be in $T_{d}$. Therefore

(*): If $d_{t} \in T_{d}$ then $d_{t a} \notin T_{d}$ if and only if $d$ and all of the symbols in any geodesic representative of $t$ are adjacent to $a$ in $\Gamma$.

For each $a \in L$ and $d_{t} \in T_{d}$, define another endomorphism $\alpha_{a^{-1}}: F \rightarrow F$ by replacing $a$ by $a^{-1}$ in Equation 7 Then

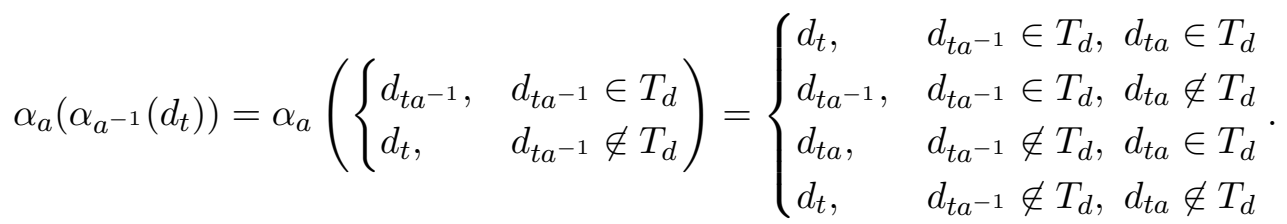

As a consequence of $(*)$ from the previous paragraph, for every $a \in L, d \in D$, and $d_{t} \in T_{d}$, we have $d_{t a} \in T_{d}$ if and only if $d_{t a^{-1}} \in T_{d}$, and so the middle two cases in last expression of the equation above cannot occur. Therefore $\alpha_{a}\left(\alpha_{a^{-1}}\left(d_{t}\right)\right)=d_{t}$, and similarly $\alpha_{a^{-1}}\left(\alpha_{a}\left(d_{t}\right)\right)=d_{t}$. Thus the maps $\alpha_{a}$ and $\alpha_{a^{-1}}$ are automorphisms of $F$ which are inverse to each other.

Finally, for each $a, b \in L$ that are adjacent in $\Gamma$ and each $d \in D$ and $d_{t} \in$ $T_{d}$, the equivalence in $(*)$ shows that the condition $d_{t a b} \in T_{d}$ is equivalent to the conjunction of the conditions $d_{t a} \in T_{d}$ and $d_{t b} \in T_{d}$, so we have

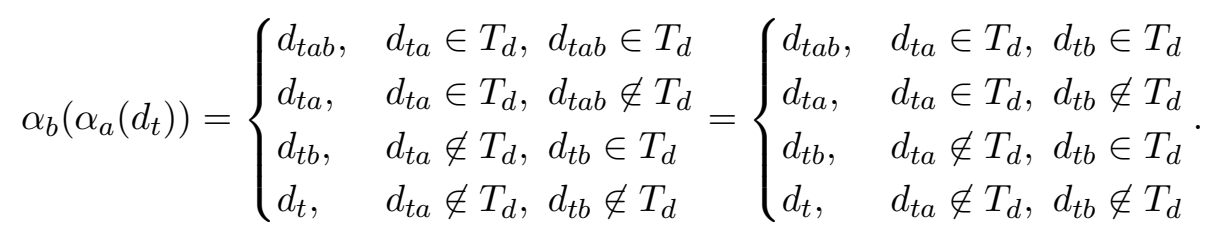

Therefore, by symmetry, $\alpha_{b}\left(\alpha_{a}\left(d_{t}\right)\right)=\alpha_{a}\left(\alpha_{b}\left(d_{t}\right)\right)$. Thus $\alpha_{a} \alpha_{b}=\alpha_{b} \alpha_{a}$ whenever $a$ and $b$ are adjacent in $\Gamma$, which implies that Equation (17) defines a homomorphism $\alpha: A \Gamma_{L} \rightarrow A u t(F)$, given by $a \mapsto \alpha_{a}$, and an action of $A \Gamma_{L}$ on the free group $F$.

Let $G:=F \rtimes A \Gamma_{L}$ be the semidirect product defined by this action. Next we show that $G \cong A \Gamma$. A presentation for $G$ is given by

$$
G=\left\langle L \cup\left(\cup_{d \in D} T_{d}\right) \mid R_{L} \cup\left(\cup_{d \in D} R_{d}\right)\right\rangle,
$$


where $R_{L}$ is the set of commutation relations defining $A \Gamma_{L}$ (induced by the edges of $\Gamma_{L}$ ) and for each $d \in D$,

$$
R_{d}:=\left\{d_{t}^{a}=d_{t a} \mid d_{t} \in T_{d}, d_{t a} \in T_{d}\right\} \cup\left\{d_{t}^{a}=d_{t} \mid d_{t} \in T_{d}, d_{t a} \notin T_{d}\right\} .
$$

Next apply Tietze transformations to simplify this presentation. Given an element $d_{t} \in T_{d}$, let $\eta_{t}$ be a geodesic representative of $t$. For each prefix $u$ of $\eta_{t}$, then $d_{\bar{u}} \in T_{d}$ as well, so the relations of the type $d_{t}^{a}=d_{t a}$ in $R_{d}$ can be used to show that $d_{t}=d_{1}^{\eta_{t}}$ in $G$. For any other geodesic representative $w$ of $t \in A \Gamma_{L}$, the relation $d_{1}^{\eta_{t}}=d_{1}^{w}$ is a consequence of the relations in $R_{L}$. If we denote $d=d_{1}$ for $d \in D$, then the presentation of $G$ is Tietze equivalent to

$$
\left\langle L \cup D \mid R_{L} \cup\left(\cup_{d \in D} R_{d}^{\prime}\right)\right\rangle,
$$

where

$$
R_{d}^{\prime}:=\left\{d^{t a}=d^{t} \mid d_{t} \in T_{d}, d_{t a} \notin T_{d}\right\} .
$$

Note that the relation $d^{a}=d$ occurs in $R_{d}^{\prime}$ if $d_{a} \notin T_{d}$, and $d_{a} \notin T_{d}$ if and only if $a$ is adjacent to $d$ in $\Gamma$. Thus the relations in $R_{d}^{\prime}$ include all the defining relations in $A \Gamma$ involving $d$. For each relation $d^{t a}=d^{t}$ in $R_{d}^{\prime}$ with $t$ a nontrivial element of $A \Gamma_{L}$, we have $d_{t a} \notin T_{d}$, which implies by $(*)$ that $a$ is adjacent to $d$ and to all of the symbols in any geodesic for $t$. This shows that the relation $d^{t a}=d^{t}$ is a consequence of the relation $d^{a}=d$ and the relations in $R_{L}$. Thus the presentation for $G$ is Tietze equivalent to

$$
\left\langle L \cup D \mid R_{L} \cup\left(\cup_{d \in D} R_{d}^{\prime \prime}\right)\right\rangle,
$$

where

$$
R_{d}^{\prime \prime}:=\left\{d^{a}=d \mid d_{a} \notin T_{d}\right\}=\left\{d^{a}=d \mid a \text { is adjacent to } d \text { in } \Gamma\right\},
$$

which is exactly the defining presentation of $A \Gamma$.

Therefore $A \Gamma \cong G=F \rtimes A \Gamma_{L}$. By induction $A \Gamma_{L}$ has a poly-free tower of length $\operatorname{chr}(\Gamma)-1$, so the proof of Proposition 2.3 completes the proof that $A \Gamma$ has a poly-free tower of length $\operatorname{chr}(\Gamma)$ and hence $\operatorname{pfl}(A \Gamma) \leq \operatorname{chr}(\Gamma)$.

Next consider the lower bound on the poly-free length. Let $m=\operatorname{clq}(\Gamma)$ and let $\widetilde{\Gamma}$ be a clique of $\Gamma$ with $m$ vertices. Then $\widetilde{\Gamma}$ is a complete graph, and the subgroup $A \widetilde{\Gamma}$ corresponding to $\widetilde{\Gamma}$ is isomorphic to $\mathbb{Z}^{m}$. Lemma 3.4 says that $m=\operatorname{pfl}(A \widetilde{\Gamma})$, and Proposition 2.2 shows that $\operatorname{pfl}(A \widetilde{\Gamma}) \leq \operatorname{pfl}(A \Gamma)$.

\section{Poly-freeness of Length 2}

In this section we prove converse of the main result in Section 3, that every graph with the doubly breakable cycle property induces a poly-free rightangled Artin group of length 2. Together with the main result in Section 3 . this completes the proof of Theorem B

Given a graph $\Gamma$ together with a corresponding set $D$ for which $\Gamma$ has the doubly breakable cycle property, let $L:=V(\Gamma)-D$ and let $\Gamma_{L}$ be the full subgraph of $\Gamma$ induced by $L$. Denote by $F(C)$ the free group on the set of connected components of $\Gamma_{L}$. If we define a homomorphism $\phi: A \Gamma \rightarrow F(C)$ by $\phi(d)=1$ for $d \in D$ and $\phi(y)=c$ whenever $y \in L$ and $c$ is the generator 
of $F(C)$ corresponding to the component of $\Gamma_{L}$ containing $y$, then $D$ is the set of dead vertices and $\Gamma_{L}$ is the living subgraph associated to this homomorphism. In the proof below, our approach follows the same lines as the proof of Theorem $\mathrm{A}$. We construct a free group $F$ (isomorphic to $\operatorname{ker}(\phi))$ and an action of $F(C)$ on $F$, in order to show explicitly that $A \Gamma$ is isomorphic to a semidirect product $F \rtimes F(C)$ of two free groups.

Proposition 6.1. If $\Gamma$ is a graph with the doubly breakable cycle property, then the right-angled Artin group $A \Gamma$ is poly-free of length 2.

Proof. Fix a set $D$ of independent vertices in $\Gamma$ such that every cycle in $\Gamma$ meets $D$ at least twice. Let $L$ be the complementary set of vertices in $\Gamma$ and let $\Gamma_{L}$ be the full subgraph of $\Gamma$ induced by $L$. Each of the connected components of the graph $\Gamma_{L}$ is a tree. Select one vertex from each component of $\Gamma_{L}$, and denote the set of these vertices by $C$. Define $F(C):=A \Gamma_{C}$ to be the subgroup of $A \Gamma$ corresponding to the subgraph $\Gamma_{C}$ induced by $C$. Since $\Gamma_{C}$ is totally disconnected, the group $F(C)$ is also the free group on $C$.

For every vertex $y \in L$, there exists a unique element $c \in C$ such that $y$ and $c$ are in the same component of $\Gamma_{L}$, and since this component is a tree, there exists a unique vertex path $\left(y^{(n)}, \ldots, y^{(2)}, y^{(1)}, c\right)$ connecting $y=y^{(n)}$ and $c$ that lies inside the component of $c$ and is of minimal length. We call $c$ the component representative of $y$ and denote it by $r_{y}$. For each $d \in D$, let $N_{d}$ denote the set of vertices in $\Gamma$ adjacent to (i.e. neighbors of) $d$ and let $\mathcal{R} N_{d}$ be the set of component representatives of the vertices in $N_{d}$. For every element $c$ in $\mathcal{R} N_{d}$ there exists a vertex $y \in N_{d}$ that is contained in the connected component containing $c$; if $y \neq c$, denote this neighbor of $d$ by $x(d, c)$. For example, for the graph in Figure $1 r_{y_{i, j}}=c_{j}$, for all $i$ and $j$. The element $x(d, c)$ is defined only in the following two cases: $x\left(d_{1}, c_{2}\right)=y_{4,2}$ and $x\left(d_{2}, c_{2}\right)=y_{3,2}$.

In the following, the normal form of an element $t \in F(C)$ refers to the freely reduced word over $C^{ \pm 1}$ corresponding to $t$. Define $X:=L-C$. For each $x \in X$, let $\mathbf{x}$ be a copy of $x$ and let $\mathbf{X}=\{\mathbf{x} \mid x \in X\}$ be the set of such copies. For each $\mathbf{x} \in \mathbf{X}$, define a set of symbols

$$
\begin{aligned}
& T_{\mathbf{x}}:=\left\{\mathbf{x}_{t} \mid t \in F(C) \text {, the normal form of } t\right. \text { does not } \\
& \text { start with a letter in } \left.\left\{r_{x}^{ \pm 1}\right\}\right\} \text {. }
\end{aligned}
$$

For each $d \in D$, define a set of symbols

$$
\begin{aligned}
& T_{d}:=\left\{d_{t} \mid t \in F(C) \text {, the normal form of } t\right. \text { does not } \\
& \text { start with a letter in } \left.\mathcal{R} N_{d}^{ \pm 1}\right\} \text {. }
\end{aligned}
$$

For any $z \in \mathbf{X} \cup D$, let $F\left(T_{z}\right)$ be the free group over $T_{z}$ and let $F$ be the free group

$$
F:=\left(*_{\mathbf{x} \in \mathbf{X}} F\left(T_{\mathbf{x}}\right)\right) *\left(*_{d \in D} F\left(T_{d}\right)\right) .
$$


Given any $c \in C$, define an endomorphism $\alpha_{c}$ of the free group $F$ by defining $\alpha_{c}$ on the generators of $F$ as

$$
\alpha_{c}\left(\mathbf{x}_{t}\right):= \begin{cases}\mathbf{x}_{t c}, & t \neq 1 \text { or } c \neq r_{x} \\ {\left[\left(\mathbf{x}_{1}^{(n-1)}\right)^{-1} \mathbf{x}_{1}^{(n)}\right] \cdots\left[\left(\mathbf{x}_{1}^{(2)}\right)^{-1} \mathbf{x}_{1}^{(3)}\right]\left[\left(\mathbf{x}_{1}^{(1)}\right)^{-1} \mathbf{x}_{1}^{(2)}\right] \mathbf{x}_{1}^{(1)},} & t=1 \text { and } c=r_{x}\end{cases}
$$

for $\mathbf{x} \in \mathbf{X}$ and $t \in T_{\mathbf{x}}$, where $\left(x^{(n)}, \ldots, x^{(2)}, x^{(1)}, c\right)$ is the minimal length path from $x$ to $c$ inside the component of $c$, and

$$
\alpha_{c}\left(d_{t}\right):= \begin{cases}d_{t c}, & t \neq 1 \text { or } c \notin \mathcal{R} N_{d} \\ d_{1}, & t=1 \text { and } c \in \mathcal{R} N_{d} \cap N_{d} \\ d_{1}^{\mathbf{x}(d, c)_{1}}, & t=1 \text { and } c \in \mathcal{R} N_{d}-N_{d}\end{cases}
$$

for $d \in D$ and $t \in T_{d}$.

Similarly, for $c \in C$, we also define an endomorphism $\alpha_{c^{-1}}: F \rightarrow F$ by

$$
\alpha_{c^{-1}}\left(\mathbf{x}_{t}\right)=\left\{\begin{array}{ll}
\mathbf{x}_{t c^{-1}}, & t \neq 1 \text { or } c \neq r_{x} \\
\mathbf{x}_{1}^{(1)}\left[\mathbf{x}_{1}^{(2)}\left(\mathbf{x}_{1}^{(1)}\right)^{-1}\right]\left[\mathbf{x}_{1}^{(3)}\left(\mathbf{x}_{1}^{(2)}\right)^{-1}\right] \cdots\left[\mathbf{x}_{1}^{(n)}\left(\mathbf{x}_{1}^{(n-1)}\right)^{-1}\right], & t=1 \text { and } c=r_{x}
\end{array},\right.
$$

for $\mathbf{x} \in \mathbf{X}$ and $t \in T_{\mathbf{x}}$, where $\left(x^{(n)}, \ldots, x^{(2)}, x^{(1)}, c\right)$ is the minimal length path from $x$ to $c$ inside the component of $c$, and

$$
\alpha_{c^{-1}}\left(d_{t}\right)= \begin{cases}d_{t c^{-1}}, & t \neq 1 \text { or } c \notin \mathcal{R} N_{d} \\ d_{1}, & t=1 \text { and } c \in \mathcal{R} N_{d} \cap N_{d}, \\ d_{1}^{\left[\alpha_{c^{-1}}\left(\mathbf{x}(d, c)_{1}\right)\right]^{-1}}, & t=1 \text { and } c \in \mathcal{R} N_{d}-N_{d}\end{cases}
$$

for $d \in D$ and $t \in T_{d}$.

As in the proof of Theorem A it is straightforward to check that the composition of the endomorphisms $\alpha_{c}$ and $\alpha_{c^{-1}}$ in either order is equal to the identity on the generating set of $F$. (The claim easily follows from $\alpha_{c}\left(x_{1}^{(i)}\left(x_{1}^{(i-1)}\right)^{-1}\right)=\left(x_{1}^{(i-1)}\right)^{-1} x_{1}^{(i)}$ and $\alpha_{c^{-1}}\left(\left(x_{1}^{(i-1)}\right)^{-1} x_{1}^{(i)}\right)=x_{1}^{(i)}\left(x_{1}^{(i-1)}\right)^{-1}$, which hold for any adjacent pair of vertices $x^{(i)}$ and $x^{(i-1)}$ in the component of $c$ at distance $i$ and $i-1$ from $c$, respectively, as measured within the component of $c$.) Therefore $\alpha_{c}$ and $\alpha_{c^{-1}}$ are mutually inverse automorphisms of $F$.

Since the group $F(C)$ is free, the map $c \mapsto \alpha_{c}$ can be extended to a homomorphism $\alpha: F(C) \rightarrow \operatorname{Aut}(F)$. Therefore (8) and (9) define an action of $F(C)$ on $F$.

Let $G:=F \rtimes F(C)$ be the associated semidirect product. A presentation for $G$ can be given by

$$
G=\left\langle C \cup\left(\cup_{\mathbf{x} \in \mathbf{X}} T_{\mathbf{x}}\right) \cup\left(\cup_{d \in D} T_{d}\right) \mid\left(\cup_{\mathbf{x} \in \mathbf{X}} R_{\mathbf{x}}\right) \cup\left(\cup_{d \in D} R_{d}\right)\right\rangle,
$$

where, for $d \in D$ and $\mathbf{x} \in \mathbf{X}$,

$$
\begin{aligned}
R_{\mathbf{x}}:= & \left\{\mathbf{x}_{t}^{c}=\mathbf{x}_{t c} \mid c \in C, \mathbf{x}_{t} \in T_{\mathbf{x}}, t \neq 1 \text { or } c \neq r_{x}\right\} \cup \\
& \left\{\mathbf{x}_{1}^{c}=\left[\left(\mathbf{x}_{1}^{(n-1)}\right)^{-1} \mathbf{x}_{1}^{(n)}\right] \cdots\left[\left(\mathbf{x}_{1}^{(2)}\right)^{-1} \mathbf{x}_{1}^{(3)}\right]\left[\left(\mathbf{x}_{1}^{(1)}\right)^{-1} \mathbf{x}_{1}^{(2)}\right] \mathbf{x}_{1}^{(1)} \mid c=r_{x}\right\},
\end{aligned}
$$


where $\left(x^{(n)}, \ldots, x^{(2)}, x^{(1)}, c\right)$ is the minimal length path from $x$ to $c$ inside the component of $c$ and

$$
\begin{aligned}
R_{d}:= & \left\{d_{t}^{c}=d_{t c} \mid c \in C, d_{t} \in T_{d}, t \neq 1 \text { or } c \notin \mathcal{R} N_{d}\right\} \cup \\
& \left\{d_{1}^{c}=d_{1} \mid c \in \mathcal{R} N_{d} \cap N_{d}\right\} \cup \\
& \left\{d_{1}^{c}=d_{1}^{\mathbf{x}(d, c)_{1}} \mid c \in \mathcal{R} N_{d}-N_{d}\right\} .
\end{aligned}
$$

Next apply Tietze transformations to simplify this presentation.

First note that if $\mathbf{x}_{t} \in T_{\mathbf{x}}$, then every prefix $u$ of the normal form of $t$ does not start with a letter in $\left\{r_{x}^{ \pm 1}\right\}$, so $\mathbf{x}_{\bar{u}} \in T_{\mathbf{x}}$ as well, and similarly for $d_{t} \in T_{d}$. Using this fact and repeatedly applying the relations of the type $\mathbf{x}_{t}^{c}=\mathbf{x}_{t c}$ and $d_{t}^{c}=d_{t c}$ in $R_{\mathbf{x}}$ and $R_{d}$, respectively, shows that in $G$ we have $\mathbf{x}_{t}=\mathbf{x}_{1}^{t}$ for all $t \in T_{\mathbf{x}}$ and $d_{t}=d_{1}^{t}$ for all $t \in T_{d}$. If we denote $\mathbf{x}=\mathbf{x}_{1}$ and $d=d_{1}$ for each $\mathbf{x} \in \mathbf{X}$ and $d \in D$, then the presentation for $G$ is Tietze equivalent to

$$
\left\langle C \cup \mathbf{X} \cup D \mid\left(\cup_{\mathbf{x} \in \mathbf{X}} R_{\mathbf{x}}^{\prime}\right) \cup\left(\cup_{d \in D} R_{d}^{\prime}\right)\right\rangle,
$$

where

$$
R_{\mathbf{x}}^{\prime}:=\left\{\mathbf{x}^{r_{x}}=\left[\left(\mathbf{x}^{(n-1)}\right)^{-1} \mathbf{x}^{(n)}\right] \cdots\left[\left(\mathbf{x}^{(2)}\right)^{-1} \mathbf{x}^{(3)}\right]\left[\left(\mathbf{x}^{(1)}\right)^{-1} \mathbf{x}^{(2)}\right] \mathbf{x}^{(1)}\right\}
$$

and

$$
R_{d}^{\prime}:=\left\{d^{c}=d \mid c \in \mathcal{R} N_{d} \cap N_{d}\right\} \cup\left\{d^{c}=d^{\mathbf{x}(d, c)} \mid c \in \mathcal{R} N_{d}-N_{d}\right\} .
$$

Second, for every $\mathbf{x} \in \mathbf{X}$ introduce a single new generator $x$ and a relation $\mathbf{x}=x^{-1} r_{x}$ in the presentation for $G$. Use these new relations to eliminate the generators $\mathbf{x} \in \mathbf{X}$ from the above presentation and obtain a Tietze equivalent presentation

$$
\left\langle C \cup X \cup D \mid\left(\cup_{x \in X} R_{x}^{\prime \prime}\right) \cup\left(\cup_{d \in D} R_{d}^{\prime \prime}\right)\right\rangle,
$$

where

$$
R_{x}^{\prime \prime}:=\left\{x^{-1} r_{x}=\left[x^{(n-1)}\left(x^{(n)}\right)^{-1}\right] \cdots\left[x^{(2)}\left(x^{(3)}\right)^{-1}\right]\left[x^{(1)}\left(x^{(2)}\right)^{-1}\right] r_{x}\left(x^{(1)}\right)^{-1}\right\}
$$

and

$$
R_{d}^{\prime \prime}:=\left\{d^{c}=d \mid c \in \mathcal{R} N_{d} \cap N_{d}\right\} \cup\left\{d=d^{x(d, c)} \mid c \in \mathcal{R} N_{d}-N_{d}\right\} .
$$

The relations in $R_{d}^{\prime \prime}$ say that each $d \in D$ commutes with all $c \in C$ and $x \in X$ that are its neighbors in $\Gamma$, just as in the standard presentation of $A \Gamma$. For each $c \in C$ and each vertex $x$ adjacent to $c$ in $\Gamma_{L}$, we have $c=r_{x}$ and $x=x^{(1)}$. The corresponding relation in $R_{x}^{\prime \prime}$ is $x^{-1} r_{x}=r_{x} x^{-1}$, which implies that $x$ and $c$ commute. When the path length in $\Gamma_{L}$ from $x$ to $c$ is 2 , with a vertex path $\left(x^{(2)}, x^{(1)}, c\right)$ from $x=x^{(2)}$ to $c=r_{x}$, the corresponding relation is $x^{-1} r_{x}=x^{(1)} x^{-1} r_{x}\left(x^{(1)}\right)^{-1}$. Since $x^{(1)}$ and $r_{x}$ commute, this implies that $x$ and $x^{(1)}$ commute. Continuing in the same fashion we see that all such relations together imply that each generator $x$ whose distance to $r_{x}$ in $\Gamma_{L}$ is $n$ commutes with the generator that is the neighbor of $x$ on the unique length minimal path from $x$ to $c=r_{x}$ inside the component of $r_{x}$. Thus, the standard relations in $A \Gamma$ can be 
recreated from the relations in $R_{x}^{\prime \prime}$ and $R_{d}^{\prime \prime}$. Conversely, each relation $x^{-1} r_{x}=$ $\left[x^{(n-1)}\left(x^{(n)}\right)^{-1}\right] \cdots\left[x^{(2)}\left(x^{(3)}\right)^{-1}\right]\left[x^{(1)}\left(x^{(2)}\right)^{-1}\right] r_{x}\left(x^{(1)}\right)^{-1}$ in $R_{x}^{\prime \prime}$ is a corollary of the defining relations in $A \Gamma$. Thus the last presentation above is Tietze equivalent to the standard presentation of $A \Gamma$.

Therefore $A \Gamma \cong G=F \rtimes F(C)$ has poly-free length at most 2 . The doubly breakable cycle property implies that $\Gamma$ is not totally disconnected, so the group $A \Gamma$ contains a $\mathbb{Z}^{2}$ subgroup and cannot be free. Thus the poly-free length of $A \Gamma$ is exactly 2.

The free group automorphisms $\alpha_{a}$ constructed in the proof of Theorem $\mathrm{A}$ permute the basis elements of the free group. Although the free group automorphism $\alpha_{c}$ of $F$ in the proof above does not have the same property, the automorphism of $F^{a b}=F /[F, F]$ induced by $\alpha_{c}$ permutes the basis elements of this free abelian group.

We conclude with a fully worked example illustrating a length 2 poly-free structure of a right-angled Artin group defined by a graph with the doubly breakable cycle property, following the proof of Theorem B. Let $\Gamma$ be the

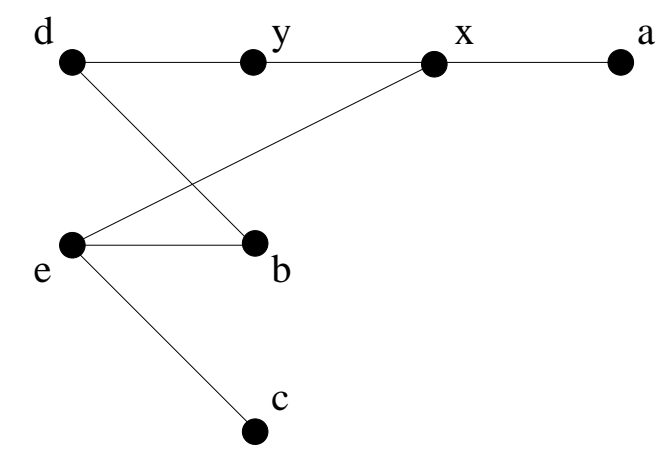

FiguRE 3. Graph with the doubly breakable cycle property

graph in Figure 3. Set $D:=\{d, e\}$. The living subgraph has 3 components and the chosen representatives are the elements of $C=\{a, b, c\}$. The only vertices in $X=L-C$ are $x$ and $y$. Denote $F_{q}:=F(a, b, c)$. We have

$$
\begin{aligned}
& T_{d}=\left\{d_{1}\right\} \cup\left\{d_{t} \mid t \in F_{q}, \text { the normal form of } t \text { starts with } c^{ \pm 1}\right\}, \\
& T_{e}=\left\{e_{1}\right\},
\end{aligned}
$$

and, for $\mathbf{z} \in\{\mathbf{x}, \mathbf{y}\}$,

$$
T_{\mathbf{z}}=\left\{\mathbf{z}_{1}\right\} \cup\left\{\mathbf{z}_{t} \mid t \in F_{q} \text {, the normal form of } t \text { starts with } b^{ \pm 1} \text { or } c^{ \pm 1}\right\} .
$$

Denote $F_{k}:=F\left(T_{d} \cup T_{e} \cup T_{\mathbf{x}} \cup T_{\mathbf{y}}\right)$. Then $A \Gamma=F_{k} \rtimes F_{q}$, where the action of $F_{q}$ on $F_{k}$ is given by the following table. 


\begin{tabular}{c|ccc} 
& $a$ & $b$ & $c$ \\
\hline$d_{1}$ & $d_{1}^{\mathbf{y}_{1}}$ & $d_{1}$ & $d_{c}$ \\
$d_{t}$ & $d_{t a}$ & $d_{t b}$ & $d_{t c}$ \\
$e_{1}$ & $e_{1}^{\mathbf{x}_{1}}$ & $e_{1}$ & $e_{1}$ \\
$\mathbf{x}_{1}$ & $\mathbf{x}_{1}$ & $\mathbf{x}_{b}$ & $\mathbf{x}_{c}$ \\
$\mathbf{x}_{t}$ & $\mathbf{x}_{t a}$ & $\mathbf{x}_{t b}$ & $\mathbf{x}_{t c}$ \\
$\mathbf{y}_{1}$ & $\left(\mathbf{x}_{1}\right)^{-1} \mathbf{y}_{1} \mathbf{x}_{1}$ & $\mathbf{y}_{b}$ & $\mathbf{y}_{c}$ \\
$\mathbf{y}_{t}$ & $\mathbf{y}_{t a}$ & $\mathbf{y}_{t b}$ & $\mathbf{y}_{t c}$
\end{tabular}

In this table, the entry in the row labeled on the left by the letter $\sigma$ and column labeled above by $\tau$ is the conjugate $\sigma^{\tau}$. In the leftmost column of the table, the $d_{t}, \mathbf{x}_{t}$, and $\mathbf{y}_{t}$ entries range over all symbols in $T_{d} \backslash\left\{d_{1}\right\}$, $T_{\mathbf{x}} \backslash\left\{\mathbf{x}_{1}\right\}$, and $T_{\mathbf{y}} \backslash\left\{\mathbf{y}_{1}\right\}$, respectively.

\section{REFERENCES}

[1] E. Artin. Theory of braids. Ann. of Math. (2), 48:101-126, 1947.

[2] Mladen Bestvina. Non-positively curved aspects of Artin groups of finite type. Geom. Topol., 3:269-302 (electronic), 1999.

[3] Mladen Bestvina and Noel Brady. Morse theory and finiteness properties of groups. Invent. Math., 129(3):445-470, 1997.

[4] Egbert Brieskorn. Sur les groupes de tresses [d'après V. I. Arnol'd]. In Séminaire Bourbaki, 24ème année (1971/1972), Exp. No. 401, pages 21-44. Lecture Notes in Math., Vol. 317. Springer, Berlin, 1973.

[5] Kenneth S. Brown. Cohomology of groups, volume 87 of Graduate Texts in Mathematics. Springer-Verlag, New York, 1982.

[6] Warren Dicks. An exact sequence for rings of polynomials in partly commuting indeterminates. J. Pure Appl. Algebra, 22(3):215-228, 1981.

[7] Carl Droms. Graph groups, coherence, and three-manifolds. J. Algebra, 106(2):484489, 1987.

[8] G. L. Fel'dman. The homological dimension of group algebras of solvable groups. Izv. Akad. Nauk SSSR Ser. Mat., 35:1225-1236, 1971.

[9] Elizabeth Green. Graph products of groups. Thesis, The University of Leeds, (1990).

[10] Susan Hermiller and John Meier. Algorithms and geometry for graph products of groups. J. Algebra, 171(1):230-257, 1995.

[11] Susan M. Hermiller and John Meier. Artin groups, rewriting systems and threemanifolds. J. Pure Appl. Algebra, 136(2):141-156, 1999.

[12] James Howie. Bestvina-Brady groups and the plus construction. Math. Proc. Cambridge Philos. Soc., 127(3):487-493, 1999.

[13] Roger C. Lyndon and Paul E. Schupp. Combinatorial group theory. Springer-Verlag, Berlin, 1977. Ergebnisse der Mathematik und ihrer Grenzgebiete, Band 89.

[14] David Meier. On the homological dimension of poly-locally free groups. J. London Math. Soc. (2), 22(3):449-459, 1980.

[15] David Meier. On polyfree groups. Illinois J. Math., 28(3):437-443, 1984. 
[16] John Meier and Leonard VanWyk. The Bieri-Neumann-Strebel invariants for graph groups. Proc. London Math. Soc. (3), 71(2):263-280, 1995.

Dept. of Mathematics, University of Nebraska, Lincoln, NE 68588-0130

E-mail address: smh@math.unl.edu

Dept. of Mathematics, Texas A\&M University, College Station, TX 778433368

E-mail address: sunik@math.tamu.edu 